\title{
An Application of ANN Model with Bayesian Regularization Learning Algorithm for Computing the Operating Frequency of C-Shaped Patch Antennas
}

\author{
Ahmet Kayabasi ${ }^{1 *}$, Ali Akdagli ${ }^{2}$ \\ ${ }^{1}$ Engineering Faculty, Department of Electrical-Electronics Engineering, Karamanoglu Mehmetbey University, 70100, Karaman, \\ Turkey \\ ${ }^{2}$ Engineering Faculty, Department of Electrical-Electronics Engineering, Mersin University, Ciftlikkoy, Yenisehir, 33343, Mersin, \\ Turkey
}

\begin{tabular}{l} 
A R T I C L E I N F O \\
\hline Article history: \\
Received: 09 August, 2016 \\
Accepted: 21 September, 2016 \\
Online: 27 October, 2016 \\
\hline
\end{tabular}

Keywords:

Patch antenna

C-shaped patch antenna

Operating frequency

Artificial neural network

Bayesian regularization

\begin{abstract}
A B S T R A C T
In this paper, an application of artificial neural network (ANN) using bayesian regularization (BR) learning algorithm based on multilayer perceptron (MLP) model is presented for computing the operating frequency of $C$-shaped patch antennas (CPAs) in $U H F$ band. Firstly, the operating frequencies of 144 CPAs having varied dimensions and electrical parameters were simulated by the XFDTD software package based on the finitedifference time domain (FDTD) method in order to generate the data set for the training and testing processes of the ANN-BR model. Then ANN-BR model was built with data set and while 129 simulated CPAs and remaining 15 simulated CPAs were employed for ANN$B R$ model training and testing respectively. In order to demonstrate its validity and accuracy, the proposed ANN-BR model was also tested over the simulation data given in the literature. The obtained results show that ANN-BR technique can be successfully used to compute the operating frequency of CPAs without involving any sophisticated methods.
\end{abstract}

\section{Introduction}

In the present age of the wireless communication systems are moving towards the miniaturization very rapidly. The patch antennas (PAs) have become popular in wireless communication technology due to their attractive features of low cost, low profile, easy production and conformability to mounting host [1]. By using the substrate materials with high dielectric constant, the smaller antennas can be achieved but this gives rise to decrease the bandwidth and efficiency performances [2]. C-shaped patch antennas (CPAs) formed by slot-loading technique are widely used owing to having better characteristics such as wideband and miniaturized structure [3-5]. In analysis of the traditional PAs techniques such as cavity model [6] and transmission line model [7] are used. However, irregular shaped PAs may not be analyzed with use of these techniques. Simulation and experimental studies are therefore, carried out in analysis and design of irregular shaped

\footnotetext{
*Corresponding Author: Ahmet Kayabasi, Engineering Faculty, Department of Electrical-Electronics Engineering, Karamanoglu Mehmetbey University, 70100, Karaman, Turkey

Tel: +905337263996

Email: ahmetkavabasi@kmu.edu.tr www.astes].com
}

https://dx.doi.org/10.25046/aj010501
PAs, in general. Powerful simulation tools, which employ electromagnetic methods involving rigorous mathematical formulation and extensive numerical procedures such as finite difference time domain (FDTD) method [8] and method of moment (MoM) [9] are widely utilized; however, the design procedure may be highly time consuming using these tools.

It is well known that current advancements in wireless communication technology have led to increase the use of PAs; hence, simple models should be utilized to analyze their performances such as bandwidth and operating frequency. On the other hand, the operating frequency is of crucial importance in the PA design process because these antennas inherently suffer from the narrow bandwidth. Alternative simple ways should therefore be investigated by taking into consideration that the analysis of the PA is a complex problem because of the fringing fields at the edges. There exist several approaches which vary in accuracy and computational efforts have been proposed to analyze and design PAs. The most widely used can be listed as formulation methods [3-5] and artificial intelligent systems (AIs) [10-15]. Formulation methods are commonly derived with the aid of the optimization 


\section{K. Ahmet et al. / Advances in Science, Technology and Engineering Systems Journal Vol. 1, No. 5, 1-5 (2016)}

algorithm such as genetic, particle swarm, differential evolution etc. The most well-known artificial intelligent systems are the artificial neural network (ANN) [10-13] and the adaptive neurofuzzy interference system (ANFIS) [14] and the support vector machine (SVM) [15].

The ANN is a mathematical model inspired by brain's structure. It is an artificial solution to complex and high nonlinear problems. The ANN mimics the working mechanism of the human brain in which highly interconnected neurons and organized into different layers. The neurons contain non-linear type of functions connected mutually by similar synaptic weights. The synaptic weights are weakened or strengthened during the learning process thanks to the learning algorithms such as Levenberg Marquardt (LM), Bayesian regularization (BR), cyclical order incremental update (COIU), Powel-Beale conjugate gradient (PBCG), Fletcher-Powel conjugate gradient (FPCG), Polak-Ribiere conjugate gradient (PRCG), one step secant (OSS) and scaled conjugate gradient (SCG). The performances of the learning algorithms highly depend on the problem, and they should be considered with their own benefits and limitations. BR learning algorithm uptades the weight and bias values according to Levenberg-Marquartd optimization. It minimizes a combination of squared errors and weights and then determines the correct combination so as to produce a network that generalizes well [10].

In our previous works [4-5], a number of approximate formulas with their own simplicity and accuracy have been proposed for calculating the operating frequencies of CPAs. In these formulas, the operating frequency calculation was based on the use of the resonant length equations together with the edge extension dimension and effective relative dielectric constant expressions proposed for rectangular PA (RPA), which leads to more complex the computing process. Several works related to ANN for computing operating frequencies of PAs have been studied in recent years [10-13]. The methods based on ANN were proposed for determining the operating frequency of annular ring [10], E [11], H [12] and L-shaped [13] PAs in the literature. A method of ANN based on MLP model was applied to compute the operating frequencies of annular ring PAs and the constructed model was separately trained with 8 different learning algorithms [10]. The ANN model with Levenberg Marquardt learning algorithm was used to compute the operating frequencies of $\mathrm{E}, \mathrm{H}$ and $\mathrm{L}$ shaped PAs [11-13]. Also in our previous work, ANFIS model has been proposed for predicting the operating frequency of CPAS [14].

In this study, a method of feed forward back propagation (FFBP) ANN model based on multilayered perceptron (MLP) has been designed to compute the operating frequencies of CPAs. In order to create a population data for training and testing the ANN network using bayesian regularization (BR) learning algorithm, the operating frequency values of 144 CPAs operating among 0.33 - $2.92 \mathrm{GHz}$ covering the most bands of GSM, LTE, WLAN and WiMAX standards are determined by means of XFDTD simulation software based on the finite-difference time domain (FDTD) method. In order to provide the generality and stability of the ANN-BR model, the parameters of 129 randomly selected CPAs were utilized to training the models and the 15 remaining's were employed to test the accuracy of the models. The validity of the ANN-BR model is then verified through simulated results of the CPAs reported elsewhere. Furthermore, the proposed model in this study was compared other methods in the literature.

\section{Design and Simulation of CPAs}

The CPA has a slot with $l$ and $w$ dimensions in the single nonradiating sides of a rectangular patch $(L x W)$ on a substrate of height $h$ with the relative dielectric constant $\varepsilon_{r}$ overall on the ground plane, as shown in Figure 1. Slot loading on the RPA results in a decrease in operating frequency, therefore the operating frequency of the CPA can be reduced effectively.

As shown in Figure 2, in order to determine the operating frequencies, simulations using the XFDTD software package were performed for 144 CPAs with different patch dimensions and various substrate dielectric constant values, as tabulated in Table 1. In the simulations by XFDTD, source wave form was chosen as Gaussian, and the maximum cell size for meshing process was set to $0.7 \mathrm{~mm}$ in cubic region. The antennas were supposed to be fed by a coaxial cable with $50 \mathrm{ohm}$ located around $x_{0}=2(W-w) / 3$ and $y_{0}=(L-l) / 2+l$. The antennas operate over the frequency range 0.33 - $2.92 \mathrm{GHz}$ corresponding to the UHF band.
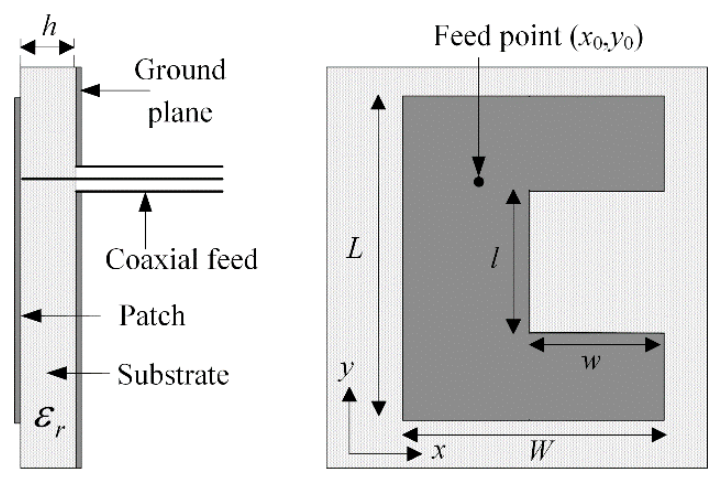

Figure 1: Geometry of CPA

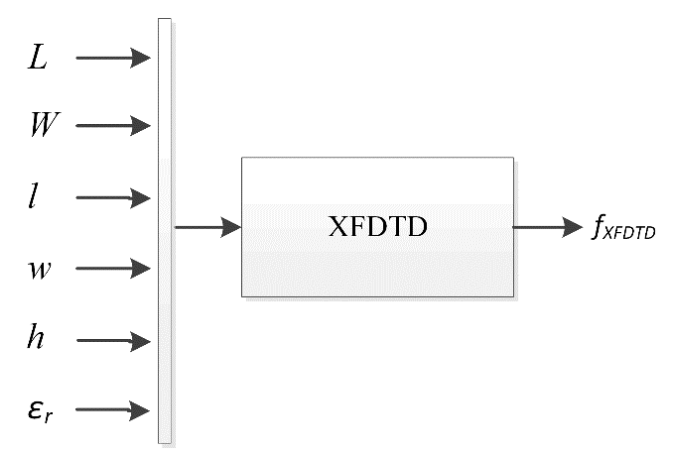

Figure 2: Simulation process by XFDTD

Table 1. The physical and electrical parameters of simulated CPAs

\begin{tabular}{ccccccc}
\hline \multicolumn{7}{c}{ Patch dimensions (mm) } \\
\cline { 2 - 6 } \\
\cline { 2 - 6 } $3 \times 4$ & $W$ & $l$ & $w$ & $h$ & $\varepsilon_{r}$ \\
\hline \multirow{3}{*}{30} & 20 & $7,12,15,20$ & $5,7,12,15$ & 1.6 & $2.33,4.28,9.8$ \\
& 60 & 40 & $13,20,30,40$ & $9,13,20,30$ & 3 & $2.33,4.28,9.8$ \\
& 90 & 60 & $20,30,40,60$ & $13,20,30,40$ & 6 & $2.33,4.28,9.8$ \\
\hline
\end{tabular}

\section{Design of the ANN-BR Model}

\subsection{Training the ANN-BR Model}

The ANN-BR model has been adapted for the computation of the operating frequency of CPAs. As shown in Figure 3, the physical and electrical parameters $\left(L, W, l, w, h\right.$ and $\left.\varepsilon_{r}\right)$ of the 


\section{K. Ahmet et al. / Advances in Science, Technology and Engineering Systems Journal Vol. 1, No. 5, 1-5 (2016)}

antennas were given as input and their respective operating frequency values were given as output for the ANN-BR network.

Thanks to the simulation data, a MLP model of ANN-BR with 3 layers of input, hidden and output layers respectively having 6, 4 and 1 neurons is designed. Whereas the computed operating frequency values are obtained as output from the model of ANNBR. While the 129 data of simulated 144 CPAs are served to train, and the remaining 15 are used to test the ANN-BR model. The proposed ANN-BR model is given in Figure 4, where $f_{X F D T D}$ and $f_{A N N-B R}$ are the operating frequencies computed by XFDTD packaged software and ANN-BR model, respectively. In the ANNBR model, "tangent sigmoid" function is used for both input and hidden layers, whereas "purelin" function is utilized for output layer. The BR learning algorithm was used in the ANN model as training algorithm, since it is capable of fast learning and good convergence. The parameters of the ANN model used in this work are tabulated in Table 2. According to (1), the value of the average percentage errors (APE) for the operating frequencies computed by the ANN-BR model was obtained as $0.687 \%$ for the 129 CPAs' training data.

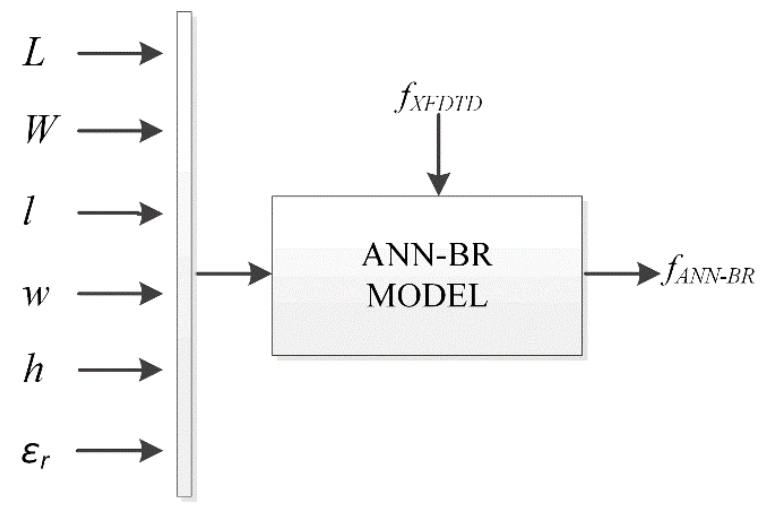

Figure 3: Training process of ANN-BR model

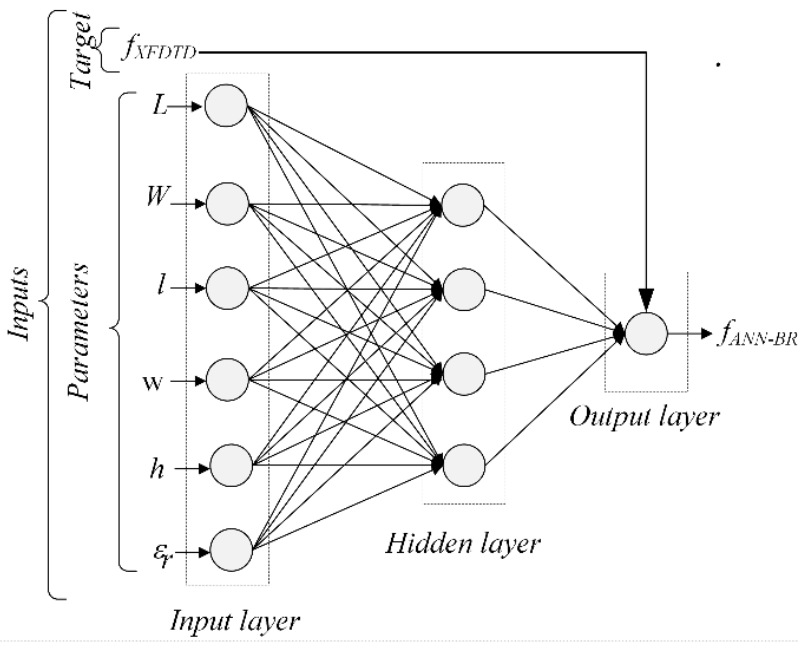

Figure 4: Block diagram of the ANN-BR model

$$
A P E=\frac{\sum\left|\frac{f_{X F D T D}-f_{A N N-B R}}{f_{X F D T D}}\right| x 100}{\text { Total antenna number }}
$$

To construct the structure of ANN-BR model, ANN toolbox of MATLAB is used. Training and testing duration take a few seconds after determined parameters that proper with our problem. In the every run process of ANN-BR model, results can be different in each run because initial weights of network is used randomly. The seed value should be fixed to get same result in the every run. For this purpose the seed in the run which is error obtained under desired value is saved. Initial weights of ANN-BR network is fixed by replacing the saved seed value in the program. This method takes time during finding the proper seed value, but after getting the proper seed value, it gives results in a few seconds.

Table 2. The parameters of ANN-BR network

\begin{tabular}{lc}
\hline Parameters & Value \\
\hline Number of input & 6 \\
Number of output & 1 \\
Epochs & 500 \\
Seed value & 2084377266 \\
Minimum gradient descent & $10^{-10}$ \\
Momentum parameter $(\mu)$ & 0.0001 \\
$\mu$ increment & 4 \\
$\mu$ decrement & 0.01 \\
Maximum $\mu$ & $10^{10}$ \\
\hline
\end{tabular}

\subsection{The Testing and Verifying the ANN-BR Model}

The remainders 15 CPAs from ones used for training process were employed for the test stage and APE value was achieved as $0.757 \%$. This process is shown in Figure 5. The computed operating frequency values and corresponding percentage errors have been given in Table 3 . It is clear from the Table 3 that our operating frequencies results are generally in very good agreement with the simulation.

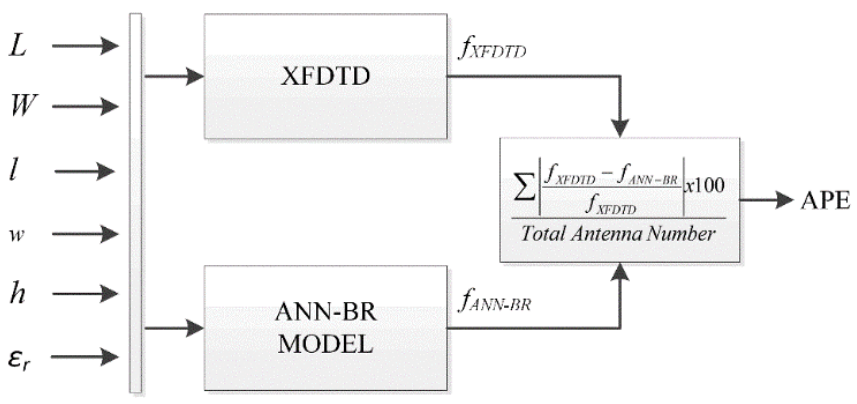

Figure 5: The test process of ANN-BR model

To demonstrate the validity and accuracy of the ANN-BR model, the model was tested against simulation data given elsewhere [3]. The test results are tabulated in Table 4. The values computed with the operating frequency formulas for CPAs given in the literature [4-5] are also given in Table 4. It was observed that the results obtained in this work are better than those predicted by other suggestions. The very good agreement between the simulated values and our computed operating frequency values supports the validity of the ANN-BR model presented here. 
Table 3. The operating frequencies determined by ANN-BR model for test process

\begin{tabular}{|c|c|c|c|c|c|c|c|c|c|}
\hline \multirow{2}{*}{$\begin{array}{l}\text { Antenna } \\
\text { number }\end{array}$} & \multicolumn{5}{|c|}{ Patch dimensions (mm) } & \multirow[b]{2}{*}{$\varepsilon r$} & \multicolumn{2}{|c|}{ Operating frequencies $(\mathrm{GHz})$} & \multirow{2}{*}{$\begin{array}{c}\text { Percentage errors } \\
(\%)\end{array}$} \\
\hline & $L$ & $W$ & $l$ & $w$ & $h$ & & Simulation & ANN-BR & \\
\hline 1 & 30 & 20 & 15 & 7 & 1.6 & 2.33 & 2.654 & 2.656 & 0.075 \\
\hline 2 & 30 & 20 & 7 & 15 & 1.6 & 4.28 & 1.380 & 1.378 & 0.145 \\
\hline 3 & 30 & 20 & 20 & 7 & 1.6 & 4.28 & 2.017 & 2.030 & 0.635 \\
\hline 4 & 30 & 20 & 12 & 15 & 1.6 & 9.80 & 0.902 & 0.895 & 0.754 \\
\hline 5 & 30 & 20 & 20 & 15 & 1.6 & 9.80 & 0.956 & 0.951 & 0.523 \\
\hline 6 & 60 & 40 & 30 & 20 & 3 & 2.33 & 1.164 & 1.157 & 0.601 \\
\hline 7 & 60 & 40 & 20 & 9 & 3 & 4.28 & 1.081 & 1.072 & 0.805 \\
\hline 8 & 60 & 40 & 40 & 20 & 3 & 4.28 & 0.887 & 0.911 & 2.649 \\
\hline 9 & 60 & 40 & 30 & 9 & 3 & 9.80 & 0.721 & 0.719 & 0.333 \\
\hline 10 & 60 & 40 & 40 & 30 & 3 & 9.80 & 0.471 & 0.469 & 0.425 \\
\hline 11 & 90 & 60 & 20 & 13 & 6 & 2.33 & 0.970 & 0.980 & 1.031 \\
\hline 12 & 90 & 60 & 40 & 30 & 6 & 2.33 & 0.776 & 0.785 & 1.198 \\
\hline 13 & 90 & 60 & 20 & 40 & 6 & 4.28 & 0.527 & 0.523 & 0.816 \\
\hline 14 & 90 & 60 & 60 & 40 & 6 & 4.28 & 0.527 & 0.524 & 0.569 \\
\hline 15 & 90 & 60 & 60 & 13 & 6 & 9.80 & 0.499 & 0.495 & 0.802 \\
\hline APE & & & & & & & & & 0.757 \\
\hline
\end{tabular}

Table 4. The comparative results for simulated CPAs in the literature [3]

\begin{tabular}{|c|c|c|c|c|c|c|c|c|c|c|c|c|c|c|c|}
\hline \multirow{4}{*}{$\begin{array}{c}\text { Antenna } \\
\text { number }\end{array}$} & \multirow{2}{*}{\multicolumn{2}{|c|}{$\begin{array}{c}\text { Slot } \\
\text { dimensions } \\
(\mathrm{mm})\end{array}$}} & \multicolumn{7}{|c|}{ Operating frequencies $(\mathrm{GHz})$} & & & & & & \\
\hline & & & \multirow{3}{*}{$\frac{\text { Sim. }}{[3]}$} & \multirow{3}{*}{$\begin{array}{c}\text { ANN- } \\
\text { BR } \\
\text { This } \\
\text { study }\end{array}$} & \multicolumn{5}{|c|}{ Calculated } & \multicolumn{6}{|c|}{ Percentage errors $(\%)$} \\
\hline & \multirow{2}{*}{$l$} & \multirow{2}{*}{$w$} & & & \multirow{2}{*}[5]{} & \multirow{2}{*}{ [4] } & \multicolumn{3}{|c|}{$[3]$} & \multirow{2}{*}{$\begin{array}{l}\text { This } \\
\text { Study }\end{array}$} & \multirow{2}{*}[5]{} & \multirow{2}{*}[4]{} & \multicolumn{3}{|c|}{ [3] } \\
\hline & & & & & & & For.1 & For.2 & For.3 & & & & For.1 & For. 2 & For.3 \\
\hline 1 & 5 & 5 & 1.562 & 1.61 & 1.562 & 1.657 & 1.502 & 1.63 & - & 3.073 & 0 & 6.082 & 3.841 & 4.353 & - \\
\hline 2 & 10 & 10 & 1.445 & 1.462 & 1.445 & 1.497 & 1.398 & 1.408 & - & 1.204 & 1.315 & 3.599 & 3.253 & 2.561 & - \\
\hline 3 & 15 & 15 & 1.286 & 1.302 & 1.286 & 1.334 & 1.309 & 1.241 & - & 1.229 & 0.467 & 3.732 & 1.788 & 3.499 & - \\
\hline 4 & 20 & 20 & 1.13 & 1.128 & 1.13 & 1.178 & 1.231 & 1.111 & 1.002 & 0.221 & 0.531 & 4.248 & 8.938 & 1.681 & 11.33 \\
\hline 5 & 25 & 25 & 0.991 & 0.995 & 0.991 & 1.035 & 1.164 & 1.008 & 0.928 & 0.404 & 0.908 & 4.44 & 17.46 & 1.715 & 6.357 \\
\hline 6 & 40 & 30 & 0.899 & 0.905 & 0.899 & 0.924 & - & 0.893 & 0.856 & 0.623 & 1.001 & 2.781 & - & 0.667 & 4.783 \\
\hline 7 & 5 & 30 & 0.929 & 0.936 & 0.929 & 0.963 & - & 1.029 & 0.904 & 0.71 & 0.215 & 3.66 & - & 10.764 & 2.691 \\
\hline 8 & 10 & 30 & 0.887 & 0.888 & 0.887 & 0.938 & - & - & 0.896 & 0.068 & 2.706 & 5.75 & - & - & 1.015 \\
\hline 9 & 2 & 30 & 0.964 & 0.97 & 0.964 & 0.982 & - & - & 0.91 & 0.643 & 1.867 & 1.867 & - & - & 5.602 \\
\hline APE & & & & & & & & & & 0.908 & 1.001 & 1.151 & 7.055 & 3.605 & 5.296 \\
\hline
\end{tabular}

\section{Conclusion}

In this paper, an application of the ANN model which has been used BR learning algorithm is successfully implemented for the prediction of accurate operating frequency of CPAs. XFDTD simulation software based on FDTD was used to define operating frequency of 144 CPAs. ANN-BR model, physically and electrical parameters of 129 CPAs were utilized training data, 15 CPAs were utilized for the test. It was seen that computed results with ANNBR for training and test data are in a good agreement with the simulation results. The operating frequency results obtained in this study were compared with different simulated and calculated results reported in the literature. The proposed model was achieved the more accurate results as compared to those of the methods proposed in the literature. This ANN model approach is simple and fast modeling which produces more accurate results for the operating frequency of the CPAs with less computational time and least errors. The most important advantages of ANN model are accuracy and easy to implement for the engineering problems which include the high nonlinearity.

\section{References}

[1] R. Garg, P. Bhartia, I. Bahl, A. Ittipiboon, Microstrip Antenna Design Handbook, Londra, Artech House 2001.

[2] K. L. Wong, Compact and Broadband Microstrip Antennas, Wiley, Interscience, 2002.

[3] A. Deshmukh, G. Kumar, "Formulation of resonant frequency for compact rectangular microstrip antennas" Microw. Opt. Techn. Let., 49: 498-501, 2007.

[4] A. Akdagli, M. B. Bicer, S. Ermis, "A novel expression for resonant length obtained by using artificial bee colony algorithm in calculating resonant 


\section{K. Ahmet et al. / Advances in Science, Technology and Engineering Systems Journal Vol. 1, No. 5, 1-5 (2016)}

frequency of C-shaped compact microstrip antennas" Turk. J. Electr. Eng. Co., 19: 597-606, 2011.

[5] A. Toktas, A. Akdagli, M. B. Bicer, A. Kayabasi, "Simple formulas for calculating resonant frequencies of $\mathrm{C}$ and $\mathrm{H}$ shaped compact microstrip antennas obtained by using artificial bee colony algorithm" J. Electromagnet. Wave., 25: 1718-1729, 2011.

[6] W. F. Richards, Y. T. Lo, D. D. Harrisson, "An improved theory for microstrip antennas and applications', IEEE T. Antenn. Propag., 29: 38-46, 1981.

[7] K. Bhattacharyya, R. Garg, "A generalized transmission line model for microstip patches", IEE PROC-H., 132: 93-98, 1985.

[8] A. Taflove, Computational Electrodynamics: The Finite-Difference Time Domain Method, Boston: Artech House, 1995.

[9] R. F. Harrington, Field Computation by Moment Methods, IEEE Press, Piscataway: NJ, 1993.

[10] A. Akdagli, A. Kayabasi, "An Accurate Computation Method Based on Artificial Neural Networks with Different Learning Algorithms for Resonant Frequency of Annular Ring Microstrip Antennas", J. Comput. Electron., 13(5): 1014-1019, 2014.

[11] A. Kayabasi, M. B. Bicer, A. Akdagli, A. Toktas, "Computing Resonant Frequency of H-Shaped Compact Microstrip Antennas Operating at UHF Band by Using Artificial Neural Networks", J. Fac. Eng. Archit. Gazi Univ., 26(4): 833-840, 2011.

[12] A. Akdagli, A. Toktas, A. Kayabasi, İ. Develi, "An application of artificial neural network to compute the resonant frequency of E-shaped compact microstrip antennas", J. Electr. Eng., 64(5): 317-322, 2013.

[13] A. Kayabasi, A. Toktas, A. Akdagli, M. B. Bicer, D. Ustun, "Applications of ANN and ANFIS to predict the resonant frequency of L-shaped compact microstrip antennas", Appl. Comput. Electrom. 29(6): 460-469, 2014.

[14] Akdagli A., Kayabasi A., Develi İ. "Computing Resonant Frequency of C Shaped Compact Microstrip Antennas by Using ANFIS", International Int J Electron, 102(3): 407-417, 2015.

[15] A. Kayabasi, A. Akdagli, "A novel method of support vector machine to compute the resonant frequency of annular ring compact microstrip antennas", Cogent Engineering, 2(1): 6, 2015.

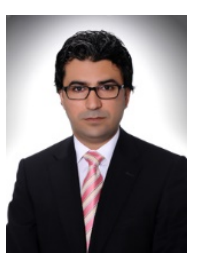

Ahmet Kayabasi was born in 1980 . He received his B.S. and M.S. degrees in Electrical and Electronics Engineering from Selcuk University, Turkey, in 2001, 2005 respectively. In 2015, he received his Ph.D. degree in Electrical and Electronics Engineering from Mersin University, Turkey. From 2001 to 2015, he was a lecturer in the Electronics and Automation Department of Silifke-Tasucu Vocational School of Selcuk University. He has been working as Assistant Professor in the Department of Electrical and Electronics Engineering at Karamanoglu Mehmetbey University. His current research interests include antennas, microstrip antennas, computational electromagnetic, artificial intelligent, and applications of optimization algorithms to electromagnetic problem such as radiation, resonance, and bandwidth. He is a regional editor member of "Advances in Science, Technology and Engineering Systems Journal (ASTESJ)"

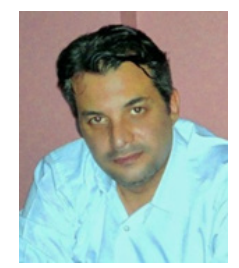

Ali Akdagli obtained the B.S., M.S. and Ph.D. degrees from Erciyes University, Kayseri, in 1995, 1997 and 2002 respectively, all in electronic engineering. From 2003 to 2006 he was an assistant professor in the electronic engineering department at Erciyes University. $\mathrm{He}$ joined the same department at Mersin University, where he currently works as a professor. He has published more than 90 papers in journals and conference proceedings. His current research interests include evolutionary optimization techniques (genetic algorithm, ant colony optimization, differential evolution, particle swarm optimization, and artificial bee colony algorithms), artificial neural networks and their applications to electromagnetic, microwave circuits, microstrip antennas and antenna pattern synthesis problems. He is an editorial board member of "Recent Patents on Electrical Engineering", International Journal of Computers and Journal of Computational Engineering. 